\title{
The relation between problem categorization and problem solving among experts and novices
}

\author{
PAMELA THIBODEAU HARDIMAN, ROBERT DUFRESNE, and JOSE P. MESTRE \\ University of Massachusetts, Amherst, Massachusetts
}

\begin{abstract}
These investigations were conducted to examine the relationship between problem-solving ability and the criteria used to decide that two classical mechanics problems would be solved similarly. We began by comparing experts and novices on a similarity judgment task and found that the experts predominantly relied on the problems' deep structures in deciding on similarity of solution, although the presence of surface-feature similarity had a clear adverse effect on performance. The novices relied predominantly on surface features, but were capable of using the problems' deep structures under certain conditions. In a second experiment, we compared groups of novices, at the same level of experience, who tended to employ different types of reasoning in making similarity judgments. Compared to novices who relied predominantly on surface features, novices who made greater use of principles tended to categorize problems similarly to how experts categorized them, as well as score higher in problem solving. These results suggest that principles play a fundamental role in the organization of conceptual and procedural knowledge for good problem solvers at all levels.
\end{abstract}

What is the relationship between problem-solving ability and the criteria one uses to decide whether or not two problems would be solved similarly? To date, attempts to answer this question have focused on investigating problem solvers at the end points of the spectrum of problem-solving skills-namely, experts and novices. For experts, the categorization of a problem as a type suggests possible solution strategies and can directly influence ability to generate a successful solution (Hayes \& H. A. Simon, 1976; Hinsley, Hayes, \& H. A. Simon, 1977; Newell \& H. A. Simon, 1972; D. P. Simon \& H. A. Simon, 1978). Research in domains such as mathematics (Schoenfeld \& Herrmann, 1982) and physics (Chi, Feltovich, \& Glaser, 1981) indicates that experts focus on the deep structures of problems (e.g., principles and concepts that could be used to solve the problems) to decide whether or not two problems would be solved similarly. These findings suggest that when attempting to solve a problem, experts begin by considering what principle or principles apply most appropriately to the situation, and then decide on a strategy or procedure that will be used to instantiate that principle, or those principles (Larkin, 1981, 1983; Larkin, McDermott, D. P. Simon, \& H. A. Simon, 1980; D. P. Simon \& H. A. Simon, 1978).

The picture is quite different for novices. When asked to categorize problems into types according to similarity

This research was supported by Grant BNS-851 1069 from the National Science Foundation. The contents do not necessarily reflect the position, policy or endorsement of NSF. We would like to thank Shari Bell for assistance in conducting Experiment 1, and William Gerace, Alice Healy, Alan Schoenfeld, and an anonymous reviewer for their insightful comments on the manuscript. Correspondence may be addressed to Pamela T. Hardiman, Department of Physics and Astronomy, University of Massachusetts, Amherst, MA 01003. of solution, novices tend to cue on surface features (e.g., problem jargon and descriptor terms) as the primary criterion of similarity (Chi et al., 1981; Schoenfeld \& Herrmann, 1982). When asked to state the general approach they would take to solve a problem, novices usually relate detailed information (e.g., equations and specific facts), rather than more general principles and concepts (Chi et al., 1981). However, as problem-solving skills develop, the reliance on deep structure to categorize problems increases (Niegemann \& Paar, 1986).

Although we can conclude that both surface features and deep structures are important, and perhaps competing, attributes of word problems that are used in judging problem similarity, it may be inappropriate to construe the use of features as being dichotomous-that is, to assume that experts use deep structures exclusively and that novices use surface features exclusively. What is clear is that the extent to which problem solvers rely on each type of information seems to be related to problem-solving ability. However, little is known about how surface features and deep structure interact in generating a problem categorization, or how problem categorization is related to problem-solving ability among novices. The two experiments we report here were conducted to investigate these issues. In Experiment 1, we designed a similarity judgment task that allowed us to examine the relative contributions of surface features and deep structure in experts' and novices' categorization decisions in the domain of classical mechanics. The results of Experiment 1 suggested that there may be individual differences in the categorization schemes used by novices. In Experiment 2, the similarity judgment task was refined in order to investigate these possible differences and to assess the relationship between categorization schemes and problemsolving ability. 


\section{EXPERIMENT 1 \\ THE INTERACTION OF SURFACE FEATURES AND DEEP STRUCTURE IN PROBLEM CATEGORIZATION}

In order to study the influence and interaction of surface features and deep structure in categorization, we designed a similarity judgment task similar to those used in studies of object categorization (Mervis, 1980; Rosch \& Mervis, 1975). In our task, a model problem and two comparison problems are presented, and the subject must decide which of the comparison problems would be solved most similarly to the model problem. The comparison problems differ in which of their attributes match those of the model problem, making it possible to investigate systematically the interaction between surface-feature and deep-structure attributes in subjects' similarity judgments. We think this type of task represents an advancement over the card-sorting task commonly used in problem categorization experiments, in which a subject sorts word problems written on index cards into several piles, which are then labeled by the subject to indicate the relationship among all the cards in a particular pile. This task requires that the subject develop a categorization scheme for all problems simultaneously. In contrast, the similarity judgment task focuses the subject's attention on specific problems, allowing problem attributes to be systematically varied. This simplifies the prediction of outcomes based on models of expert and novice performance, as well as the analysis of data and the interpretation of results.

In our similarity judgment task, a given comparison problem could match the model problem in surface features (S), deep structure (D), both surface features and deep structure (SD), or neither surface features nor deep structure $(\mathrm{N})$. These comparison problems were paired, such that one and only one problem in the pair matched the model problem in deep structure. This led to four types of pairings, which we will refer to as comparison types: (1) S-D, (2) S-SD, (3) N-D, and (4) N-SD. If it is the case that experts and novices rely primarily on different kinds of problem attributes in making similarity judgments, then the patterns of performance expected for experts and novices should differ. If one assumes that experts base their categorization decisions solely on deep structures, then they should choose the comparison problem that matches the model in deep structure $100 \%$ of the time, and select $\mathrm{D}, \mathrm{SD}, \mathrm{D}$, and $\mathrm{SD}$, respectively, for the four comparison types. Novices who base their categorization decisions solely on surface features should choose the comparison problem that matches the model problem in surface features whenever it is possible to do so. Thus, they should choose $S$ in the $S-D$ pairing and SD in the $\mathrm{N}-\mathrm{SD}$ pairing. When surface features do not allow a distinction to be made, as in S-SD and N-D, either alternative should be equally likely. Hence, novices' choices should match the model in deep structure $0 \%, 50 \%, 50 \%$, and $100 \%$ of the time for S-D, S-SD, N-D, and N-SD comparison types, respectively, and $50 \%$ of the time overall.

\section{Method}

Subjects. The novice subjects were 45 undergraduate students at the University of Massachusetts, who had completed the first semester physics course in classical mechanics and received a grade of B or better. The expert subjects were $8 \mathrm{PhD}$ physicists and two advanced physics graduate students who were nearing completion of the $\mathrm{PhD}$ requirements. The novice subjects performed both a categorization task and a problem-solving task, and were paid for their time. The expert subjects volunteered their time and only performed the categorization task.

Categorization task. Each task item on the categorization task comprised three elementary mechanics problems similar in style and level of difficulty to problems in an introductory mechanics text (i.e., Resnick \& Halliday, 1977). Each word problem was three to five lines long and contained neither pictures nor diagrams. For each item, one problem was designated as the model problem, while the other two were designated as comparison problems. The subjects were to indicate which of the two comparison problems "would be solved most similarly" to the model problem. A response was considered correct if a subject chose the comparison problem that matched the model problem in deep structure (i.e., the physical principle that would be applied to solve both problems was the same). The items were all constructed by the second author. The categorization of the comparison problems as S, D, SD, or N problems was verified by the third author and an upper level physics graduate student.

There were eight model problems, two dealing with energy principles, two dealing with momentum principles, two dealing with angular momentum principles, and two dealing with Newton's Second Law of Kinematics. Each model problem appeared four times, once with each of the four types of comparison problem pairings. This yielded 32 items composed of one model problem and two comparison problems (see Appendix 1 for a list of all the problems).

The experiment was run on IBM-compatible PCs. The subject was told to carefully read the model problem and the two comparison problems that would appear below it, and to decide which comparison problem would be solved most similarly to the model problem. The items were presented in random order, with no limit imposed on response time. Most subjects completed the task within $45 \mathrm{~min}$.

Problem-solving task. In a separate hour-long session, the novice subjects were given a problem-solving task that contained seven classical mechanics problems. Four problems required the application of one principle for solution, whereas three problems required the application of two principles. Henceforth we will only discuss performance on the single-principle problems, since few subjects were able to solve the two-principle problems. In style and level of difficulty, the single-principle problems were all similar to the problems appearing in the textbook and the problems used in the categorization task. The principles involved in the four problems were: Newton's Second Law, the conservation of energy, the conservation of linear momentum, and the conservation of angular momentum. Each problem was graded on a 10-point scale by two physicists; whenever the score on a problem differed by two or more points, the solution was discussed, and a score was determined by consensus. The total scores on the problem-solving task ranged from 1 to 40 points, with a mean of 21.2 points and a standard deviation of 11.42 points.

\section{Results}

The performances of the 45 novices and 10 experts were compared in a 2 (groups) $\times 4$ (comparison types, or pairings) $\times 8$ (model problems) analysis of variance. In 
Table 1

Predicted and Observed Percentage of Correct Responses for Experts and Novices in Experiment 1

\begin{tabular}{cccccc}
\hline & \multicolumn{2}{c}{ Experts } & & \multicolumn{2}{c}{ Novices } \\
\cline { 2 - 3 } \cline { 5 - 6 } Comparison Type & Predicted & Observed & & Predicted & Observed \\
\hline S-D & 100 & 66 & & 0 & 26 \\
S-SD & 100 & 71 & & 50 & 54 \\
N-D & 100 & 84 & & 50 & 67 \\
N-SD & 100 & 91 & & 100 & 87 \\
Total & 100 & 78 & & 50 & 59 \\
\hline
\end{tabular}

Note $-\mathrm{S}=$ surface features, $\mathrm{D}=$ deep structure, $\mathrm{SD}=$ both surface features and deep structure, $N=$ neither surface features nor deep structures.

general, the experts were better able to determine whether two problems would be solved through application of the same principle, choosing the comparison problem that matched the model problem in deep structure $78 \%$ of the time. Novices chose the deep-structure alternative 59\% of the time, which was significantly less often than the experts did $\left[F(1,53)=28.78, M S_{\mathrm{e}}=9.83, p<.0001\right]$. As predicted by our assumptions about expert and novice performance, there was a difference in how the two groups responded to the four comparison types, as indicated by the group $\times$ comparison type interaction $[F(3,159)=$ $10.00, M S_{\mathrm{e}}=1.46, p<.0001$; see Table 1 for the means]. Therefore, we will discuss the influence of comparison types for experts and novices separately.

Experts. Comparison type should have had no influence on the experts' performances if the experts had based their decisions about solution similarity strictly on deep structure (i.e., on the principles involved). However, there was a significant main effect of comparison type for experts $\left[F(3,27)=10.56, M S_{\mathrm{e}}=1.04, p=.0001\right]$, indicating that the four comparison types were not equally difficult. The mean performances for the comparison types (see Table 1) suggest that surface features have an adverse influence on experts' categorization decisions. Although the differences among these means were not all significant, they do follow the trend predicted for novices, suggesting that the experts were adversely affected by the same kinds of conditions that negatively influenced the novices. Performance on the N-SD items was significantly better than that on each of the other three comparison types $(p<.005$, Bonferroni familywise error rate: $E F / k=$ $.05 / 6=.008)$. The mean performance of the experts was significantly higher, at $p<.01$, than that of the novices for each of the four comparison types except the N-SD type, where performance was quite high for both groups (see Table 1). Thus, although experts appear to focus on deep structure to a greater degree than do novices, surface features do interfere with their performance.

Novices. Consistent with the assumption that novices cue on surface features when making similarity judgments, comparison type did influence the novices' performances $\left[F(3,132)=150.04, M S_{\mathrm{e}}=23.41, p<.0001 ;\right.$ means are in Table 1]. All pairwise comparisons differed significantly by $p<.0002(E F / k=.05 / 6=.008)$. These results indicate that surface features play a major role in novices' categorization schemes, and directly influence the process by which novices decide whether or not two problems would be solved similarly. For example, if one comparison problem matched the model problem in both surface features and deep structure, then the decision that they would be solved similarly was facilitated $(71 \%$ correct for S-SD and N-SD pairings vs. $46 \%$ correct for S-D and $\mathrm{N}-\mathrm{D}$ pairings). However, if a comparison problem matched the model problem only in surface features, then the decision that they would be solved similarly was adversely affected $(40 \%$ correct for the S-D and S-SD pairings vs. $77 \%$ correct for the N-D and N-SD pairings).

Despite their attraction to surface features as a means of judging similarity of solution, novices as a group do not seem to rely solely on surface features. For S-D iterns, in which the novices should have been most prone to ignoring deep structure, the $95 \%$ confidence interval (CI) was $20 \%<M<32 \%$, well above the predicted $0 \%$ correct had they used a strict surface-feature categorization scheme. Furthermore, in the $\mathrm{N}-\mathrm{D}$ items, where there was no distraction due to surface features, the proportion of deep-structure matches was significantly above the predicted $50 \%$ performance $(95 \% \mathrm{CI}=63 \%<M<71 \%$ ).

For both the experts and the novices, performance was influenced by model problem $\left[F(7,371)=8.46, M S_{\mathrm{e}}=\right.$ $1.58, p<.0001$; see Table 2 for means]. The items involving angular momentum were the most difficult, whereas those involving energy tended to be easier. In all but one of the eight model problems, the experts made more deep-structure judgments than did the novices, producing an interaction between model problem and group $\left[F(7,371)=3.05, M S_{\mathrm{e}}=.57, p=.0039\right.$; see Table 2]. There was also a significant interaction of model problem and comparison type $[F(21,1,113)=13.33$, $M S_{\mathrm{e}}=1.67, p<.0001$; see Table 3], suggesting that the difficulty of making a decision based on deep structure in the various conditions is related to the context of the problem.

The extent to which novices appear to rely on deep structure when making categorization decisions is related to their problem-solving ability as measured by the problem-solving task. The correlation between total categorization score and the score on the problem-solving skills test was . $30[F(1,43)=4.376, p=.0424]$. Furthermore, performance on the problem-solving task sup-

Table 2

Percentage Correct on the Eight Model Problems for the Expert and Novice Groups in Experiment 1

\begin{tabular}{|c|c|c|c|}
\hline \multirow[b]{2}{*}{ Model Problem } & \multicolumn{2}{|c|}{ Group } & \multirow[b]{2}{*}{ Overall } \\
\hline & Expert & Novice & \\
\hline 1 & 1.00 & .78 & .82 \\
\hline 2 & .80 & .43 & .50 \\
\hline 3 & .92 & .60 & .66 \\
\hline 4 & .75 & .59 & .62 \\
\hline 5 & .70 & .56 & .58 \\
\hline 6 & .65 & .52 & .54 \\
\hline 7 & .55 & .61 & .60 \\
\hline 8 & .88 & .64 & .68 \\
\hline
\end{tabular}


Table 3

Percentage of Correct Responses on the Eight Model Problems for Each of the Four Comparison Types in Experiment 1

\begin{tabular}{ccccc} 
& \multicolumn{4}{c}{ Comparison Type } \\
\cline { 2 - 5 } Model Problem & S-D & S-SD & N-D & N-SD \\
\hline 1 & .62 & .71 & .96 & .98 \\
2 & .22 & .60 & .33 & .84 \\
3 & .25 & .71 & .74 & .93 \\
4 & .33 & .29 & .89 & .96 \\
5 & .25 & .25 & .93 & .91 \\
6 & .45 & .89 & .18 & .56 \\
7 & .29 & .31 & .87 & .93 \\
8 & .27 & .82 & .71 & .93 \\
\hline
\end{tabular}

Note-S = surface features, $D=$ deep structure, $S D=$ both surface features and deep structure, $N=$ neither surface features nor deep structures.

ports the notion that the novices were better able to select deep-structure matches on the similarity judgment task in problem contexts that they understood better. More specifically, subjects displayed a poor performance in both the problem-solving task and the similarity judgment task on problems involving angular momentum, whereas they displayed a relatively good performance on problems involving energy.

\section{Discussion}

The findings of Experiment 1 are consistent with the existing literature in indicating that the ways in which subjects categorize problems in classical mechanics are related to problem-solving expertise in physics. This is most obviously reflected by the greater reliance of the experts on deep-structure similarity, and of the novices on surface-feature similarity. The experts were much more likely to judge that two problems would be solved similarly if they were similar in deep structure. In contrast, the novices often indicated that problems with similar surface features would be solved similarly. However, the likelihood that both the expert and novice subjects would select the deep-structure alternative was influenced by what other problem attributes were present in the comparison problems. Among the novices, and to some extent among the experts, this performance pattern could be interpreted in terms of a threshold-type model (Smith, Shoben, \& Rips, 1974).

If the initial perception of similarity of one of the comparison problems to the model problem was high, a threshold model would predict that the subject would be inclined to make a response on the basis of this overall impression of similarity, without conducting any further analysis. Hence, we note the relatively high rate of choosing the surface-feature alternative in the S-D and N-SD comparison types where surface features were pitted against alternatives that had no obvious superficial similarity to the model problem. If neither comparison problem succeeded in crossing the threshold of similarity (as in the N-D items), the subjects were forced to consider more carefully what would constitute similarity, and hence, they might be more likely to consider principles.
Is it clearly beneficial for novices to consider principles in categorizing problems merely because experts appear to do so? Experiment 1 suggests the answer to this question may be yes, since there was a correlation between novices' problem-solving and categorization scores. The novices as a group varied considerably in the degree to which they judged problems as similar that were matched in deep structure. The better novice problem solvers made more similarity judgments on the basis of deep structures than did the poorer novice problem solvers. Thus, in the domain of physics, the ability to categorize problems as similar that are matched in deep structure seems to be beneficial to problem solving.

In Experiment 2, we will consider the issue of individual differences among novices more carefully. In doing so, we will need to clarify what types of reasoning lead to particular categorization responses, since the binary nature of the responses required in Experiment $1 \mathrm{did}$ not make the subjects' reasoning explicit. For example, the assumption that novices who chose the deep-structure alternative did so as a result of actually considering the problems' deep structure may not be valid. On the other hand, novices may have attempted to use deep structure more often than their actual performance indicates, but may not have been able to do so correctly. Therefore, in Experiment 2, we modified the similarity judgment task in an effort to make the subjects' reasoning more explicit, as well as further to explore the relationship between problem solving and problem categorization.

\section{EXPERIMENT 2 CATEGORIZATION CRITERIA AND PROBLEM-SOLVING ABILITY OF NOVICES}

Study of the ends of the spectrum of problem-solving skill, namely experts and novices, indicates there is a relationship between categorization criteria and problemsolving ability. The relevance of this finding for understanding the development of expertise would increase if this relationship could also be demonstrated among novices at the same level of experience. Such a demonstration would indicate that skill acquisition is influenced from the beginning by the types of cues to which novices try to pay attention, and that the foundations for the acquisition of expertise are laid early in the learning process. Instruction that attempts to facilitate the use of general principles may be more effective than instruction that ignores it.

In fact, the correlation between frequencies of deepstructure decisions and problem-solving scores in Experiment 1, as well as related research (such as Silver, 1979), suggests that a more conclusive demonstration is possible. In order to demonstrate that categorization criteria and problem-solving ability were related for novices with the same level of experience, we needed a task that allowed us to examine more directly subjects' reasons for making categorization decisions. Simply inferring subjects' reasons for responses, as in Experiment 1, might 
be misleading-for example, a misidentified principle might have led to an incorrect response, even though the novice was cueing on deep structure.

Therefore, we simplified the similarity judgment task used in Experiment 1, such that there was only one comparison problem presented with the model problem in each set. This led to four comparison types: (1) S, (2) D, (3) SD, and (4) N. The comparison type nomenclature now denotes the problem attributes shared by the two problems in each item; for example, in the SD items, both problems shared surface features and deep structure. The subjects were asked to decide whether or not the two problems would be solved similarly, and to give a reason for each response. Subjects reasoning appropriately according to deep structure should have responded "no" to the $\mathrm{S}$ and $\mathrm{N}$ items, since these problems could not be solved using the same principle as the model problem, and "yes" to the D and SD items, which could be solved using the same principle as the model problem. In contrast, subjects categorizing by means of surface features should have responded "yes" to the S as well as the SD items, since the surface features matched those of the model problem, and "no" to the D and N items, which did not match the model in surface features. Hence, the responses of surface-feature users should have been correct only $50 \%$ of the time.

The reasons subjects gave for each decision provided the basis for separating the subjects into groups of different types of reasoners. This allowed us to determine whether different patterns of responses were associated with different types of reasoning.

\section{Method \\ Subjects. Forty-four undergraduate students at the University of Massachusetts who had completed a first semester classical mechanics course participated in this study. They performed the categorization task and a problem-solving task, which included a mathematics component, and were paid. They granted us permis- sion to obtain their SAT verbal and mathematics scores. To have baseline data against which to compare the novice data, 7 expert PhD physicists also performed the categorization task and were paid for their participation.}

Categorization task. The word problems used in the categorization task were similar in type and difficulty to those used in Experiment 1 (they are listed in Appendix 2). They were also written by the second author and verfied by the other two authors. Two word problems were paired for each of the 32 items, one model problem and one comparison problem of type S, D, SD, or N. There were eight model problems, each of which appeared four times, once with each of the four comparison problems. A response was considered "correct" if it was the one expected (as defined earlier) when appropriate deep-structure reasoning was used.

The task was presented in a booklet, with two problems per page. The subjects were instructed to decide whether or not the two problems would be solved similarly and to respond by stating "yes" or "no." They were then to provide a reason for their response One hour was allowed for the task, and all subjects finished within this time limit.

Each of the reasons that the subjects gave was classified blindly by the first author, P.T.H., according to the following (non-mutually exclusive) characteristics: whether it involved surface features, whether it was equation-based, whether it was physics terminology- based, and whether it involved principles. The subjects were classified into three groups on the basis of the type of reasoning most frequently employed: (1) surface-feature, (2) principle, or (3) mixed. Classification in either the surface-fearure group or the principle group meant that the subject had considered either surface features or principles on 17 or more of the 32 items. The members of the mixed group employed a variety of reasoning strategies, none of which was used a majority of the time; they commonly employed equation-based or physics terminology-based reasoning on a large proportion of the iterns. There were 17 subjects in the surface-feature group, 11 subjects in the principle group, and 16 subjects in the mixed group.

Problem-solving task. The problem-solving task contained four problems, which were the same as the single-principle problems given in Experiment 1. This task, which was a portion of a larger task assessing physics knowledge, also included a mathematics proficiency task. One hour was allotted for completion of the whole task.

Each of the four problem-solving questions was graded on a 10 -point scale. Scores ranged from 0 points to 34 points, with a mean of 12.4 points and a standard deviation of 10.28 points. The math proficiency scores ranged from 14 to 40 of a possible 40 points, with a mean of 29.5 points and a standard deviation of 6.4 points.

\section{Results}

The performances of the 44 novice and 7 expert physicists on the categorization task were compared in a 2 (groups) $\times 4$ (comparison types) $\times 8$ (model problems) ANOVA. As in Experiment 1, the experts made more correct decisions on the basis of matching deep structure (95\%) than did the novices as a group $[62 \% ;[F(1,48)=$ $\left.70.27, M S_{\mathrm{e}}=21.34, p<.0001\right]$, or any of the three novice subgroups [surface features, $56 \%$, mixed, $63 \%$, principles, $69 \% ; F(1,22)=113.35, M S_{\mathrm{e}}=24.39, p<$ $.0001, F(1,21)=131.26, M S_{\mathrm{e}}=15.76, p<.0001$, and $F(1,16)=73.63, M S_{\mathrm{e}}=9.29, p<.0001$, respectively]. However, on the average, the novices made many more decisions that were correct on the basis of deep structure than one might expect; the performance of the novice subjects, at $62 \%$ correct, was significantly higher than the $50 \%$ correct predicted for novices if we assume that they employ only surface features in categorization $(95 \% \mathrm{CI}=59 \%<M<63 \%)$.

Comparison type. For the novices, but not the experts, categorization performance was influenced by comparison type, as indicated by a 3 (novice groups) $\times 4$ (comparison types) $\times 8$ (model problems) ANOVA $[F(3,129)=$ 132.71, $M S_{\mathrm{e}}=26.79, p<.0001$ ], and a 4 (comparison types) $\times 8$ (model problems) ANOVA for experts $\left[F(3,18)=1.30, M S_{e}=.0899, p=.3061\right]$. Novice performance on each type differed from that of every other type at a level of $p<.001(E F / k=.05 / 6=.008)$. As can be seen in Table 4 , subjects experienced the most difficulty in correctly rejecting $S$ comparison problems as being appropriate matches to the model problem. This result, in combination with the high rate of correct acceptance of the SD comparison problem, supports our findings from Experiment 1 by indicating the relevance that novices attach to surface features in making decisions about solution similarity. In both experiments, the presence of surface-feature similarity depressed the rate of deep-structure decisions when it was uncorrelated with 
Table 4

Percentage of Correct Responses for the Three Novice Groups on the Four Comparison Types in Experiment 2

\begin{tabular}{cccc}
\hline & \multicolumn{3}{c}{ Novice Group } \\
\cline { 2 - 4 } Comparison Type & Surface Feature & Mixed & Principle \\
\hline S & 18 & 23 & 43 \\
D & 39 & 53 & 64 \\
SD & 76 & 87 & 78 \\
N & 90 & 90 & 91 \\
All & 56 & 63 & 69 \\
\hline
\end{tabular}

Note-S = surface features, $D=$ deep structure, $S D=$ both surface features and deep structure, $N=$ neither surface features nor deep structures.

deep-structure similarity (as in S-D and S-SD items in Experiment 1 and $S$ items in this experiment) and increased the rate when it was correlated (as in N-SD items in Experiment 1 and SD items in this experiment).

The importance of surface-feature similarity to novices does not mean that deep structure is not considered, as we suggested in Experiment 1. In Experiment 2, the novices were much better at correctly accepting D comparisons than expected (50\% actual vs. 0\% expected). When there was no competition from surface features, the subjects were much more capable of making correct decisions involving deep structures. In cases where there was neither surface-feature nor deep-structure similarity (i.e., $\mathrm{N}$ comparisons), the subjects were reasonably good at assessing the lack of similarity and making a correct rejection ( $90 \%$ correct).

Reasoning employed. As expected, the experts nearly universally ( $93 \%$ of the time) provided reasons for their judgments of similarity that were based on physics principles. The principles involved were identified correctly 98\% of the time. Clearly, experts reason primarily on the basis of deep structure, as their responses in both Experiments 1 and 2 indicate, and do so appropriately.

Novices differ from experts and from each other in the degree to which they utilize principles in their reasoning. On the average, members of the principle group mentioned principles $70 \%$ of the time, members of the mixed group did so $23 \%$ of the time, and members of the surfacefeature group did so $6 \%$ of the time, so there were major differences among the groups in propensity to employ principles in reasoning. These principles were identified correctly $60 \%, 61 \%$, and $62 \%$ of the time, respectively. Thus, when the novices chose to utilize principles in an explanation, there was no difference among the three groups in the rates of correct identification, although there were marked differences in the frequencies of using principles among the three groups.

Comparison type also influenced the frequency of principle use $\left[F(3,123)=3.09, M S_{\mathrm{e}}=.327, p=.0296\right]$, although the effect was considerably more minor than the group effect. The frequencies of principle-based explanations ranged from $30 \%$ for $\mathrm{S}$ items to $37 \%$ for $\mathrm{N}$ items (the frequencies for D and SD items were $35 \%$ and $31 \%$, respectively). These data indicate that subjects were slightly less likely to use principles in their reasoning if the model and comparison problems shared the same set of surface features. Comparison type had a much greater influence on the likelihood that correct principle-based reasoning would be used $\left[F(3,123)=13.92, M S_{\mathrm{e}}=\right.$ $1.711, p<.0001]$. Principles were used correctly least often for $S$ items (12\% of all answers used correct principle reasoning) and most often for $\mathrm{N}$ items (29\%; frequencies for D and SD items were $19 \%$ and $24 \%$, respectively). Note that correct responses for both the hardest and the easiest items ( $\mathrm{S}$ and $\mathrm{N}$ ) required that the subjects identify two different principles to draw the correct conclusion concerning the relationship between the model and comparison problems. Since essentially the same amount of work is required for these two item types, this finding suggests that surface-feature similarity obviates the comparison process, as we suggested earlier with the threshold idea.

The tendency to employ principles in reasoning is related to overall success in categorization. The surfacefeature group, with $56 \%$ correct, performed significantly lower than both the mixed group, with $63 \%$ correct $[t(31)=2.59, p=.0143]$, and the principle group, with $69 \%$ correct $[t(26)=3.95, p=.0005]$. The three groups also tended to have difficulty with different types of problems, as was indicated by an interaction of group and comparison type $\left[F(6,123)=3.08, M S_{\mathrm{e}}=.6301\right.$, $p=.0077]$. (See means in Table 4.)

As can be seen in Table 4, the relative difficulty of the four comparison types was the same for all three groups. The performances on the SD and $\mathrm{N}$ items did not differ significantly among the groups, which is consistent with the $100 \%$ correct predicted performance for all subjects. As expected, it was on the $S$ and $D$ items that the differences among the groups appeared. The surface-feature group performed lower than the principle group on both $S$ and D items $[t(26)=3.10, p=.0046$, and $t(26)=$ $3.23, p=.0033$, respectively]. We had predicted that surface-feature users would make correct responses $0 \%$ of the time on these two comparison types but that principle users would be correct $100 \%$ of the time. Thus, the performances of these groups were in the predicted directions.

Clearly, although the performance of the principle group was much better than that of the average novice, their performance was far from that predicted for one who relies on principles alone. Two factors contribute to this outcome: (1) Principles were not used in every problem analysis, and (2) the principles identified were often inappropriate. Of the $70 \%$ of the time that members of the principle group used principles, $38 \%$ of the time they identified principles incorrectly. Hence, what may be more important than the appropriateness of a principle in the development of expertise is the frequency with which one attempts to apply principles to a problem analysis.

The data argue that attempted principle use is related to problem-solving ability. Mean performances on the problem-solving task were $14 \%, 32 \%$, and $57 \%$ correct for the surface-feature, mixed, and principle groups, respectively. The three groups were significantly different on this measure $\left[F(2,41)=16.19, M S_{\mathrm{e}}=1002\right.$, $p<.0001]$, and each group differed from the other 
at a level of $p<.008$. The correlation between the frequency of attempts of an individual to reason by principle and the problem-solving test score was .63 $[F(1,42)=27.657, p<.0001]$. One might argue that a third factor, such as intelligence, is responsible for this relationship. However, even when level of mathematics proficiency (which we take as an index of quantitative ability) was partialed out, the correlation was still significant $(r=.505, Z=3.516, p<.0004)$. The correlation also holds when SAT mathematics scores are used as the index of quantitative ability $(r=.613, Z=4.503, p<.0001)$. Novices who attempt to categorize problems using principles tend to be better problem solvers.

\section{Discussion}

Experiment 2 demonstrates that the relationship between use of principles in categorization and problemsolving skills is not only an appropriate characteristic for making distinctions between experts and novices, but is appropriate for distinguishing among "good" and "poor" novice physics students with similar educational experiences. Novices who attempt to analyze mechanics problems using principles make more correct judgments concerning solution similarity and are better problem solvers. Note that these novices were often incorrect in identifying the principle needed to solve a problem, but that the principle-based approach to problem categorization generally appears to have had a value beyond the successfulness of the attempt to classify a problem.

Why is principle use so highly correlated with problemsolving ability? We believe that storing information about types of physics problems in terms of general principles, as opposed to the equations and surface features that novices generally employ (Chi et al., 1981; Mestre, Dufresne, Gerace, Hardiman, \& Touger, in press), is a much more efficient form of representing physics knowledge. The effort required to organize physics knowledge in terms of broad categories is probably initially much greater than that required to organize knowledge in terms of equations. However, the effort involved in maintaining categorical information is much less than that needed to maintain and search through a large equation database. Therefore, our findings indicate that 3 months after finishing their mechanics course, principle users could solve problems more effectively than surface-feature users could. Although this study cannot address the causal relation between principle use and problem-solving skill, it suggests that pedagogy in physics might be more effective if attempts were made to convey information in a manner conducive to organization by principles, a view supported by other research as well (Eylon \& Reif, 1984; Heller \& Reif, 1984).

\section{GENERAL DISCUSSION}

In these two studies, we attempted to characterize how and when novice physics students and expert physicists use surface features and deep structure to determine that two problems would be solved similarly, and how cate- gorization and problem-solving skills are related. In agreement with other studies (Chi et al., 1981; Schoenfeld \& Herrmann, 1982), we found the presence of surface features to adversely influence the categorization decisions of novices and of experts to some extent as well. Despite the apparent difficulty in ignoring the semblance of similarity conveyed by surface features, the conclusion that novices focus almost exclusively on surface-feature similarity is unwarranted.

Two pieces of information argue against such a conclusion. First, in conditions in which surface-feature similarity was not available to "assist" in a decision, the performance of novices was much better than what would have been expected if they had been relying solely on surface-feature similarity. Second, when the novices were asked to state why they believed two problems would be solved similarly, many of them responded with arguments based on principles, although equation-based reasoning was also fairly common.

Novices are not a uniform crowd. Some do rely primarily on surface-feature similarity to categorize problems, while others attempt to reason fairly consistently by principles. It is not at all clear that a picture of the novice as progressing from reliance on surface features to reliance on deep structure is accurate. Novices who are better problem solvers, and presumably the ones more likely to continue in the field, tend to apply principles more often when deciding whether or not two problems would be solved similarly. Surface features may interfere with the decision, but they are not the primary focus of attention for good problem solvers. The conclusion that surfacefeature similarity is of minimal importance to good novices is supported by the recent work of Chi, Bassok, Lewis, Reimann, and Glaser (1987) on the initial encoding of worked out examples of physics problems. Their work indicates that good physics students make greater numbers of explanatory statements when attempting to understand examples and that they attempt to justify their conclusions through use of the principles, concepts, and definitions introduced in the text. Poor students do not develop adequate explanations of the steps taken to solve an example problem and, hence, must make decisions more on the basis of similarities with specific problems.

These sets of results suggest that our goal as educators should be to structure the information presented in the classroom in a way that assists the learner in organizing knowledge by principles. There is evidence that the standard pedagogical practices do not incorporate this strategy (Collins, Brown, \& Newman, in press). We may not be able to ensure that every student views principles as fundamental, but the centrality of principles to both experts and the better novices suggests this is the path that is more likely to lead to eventual understanding.

\section{REFERENCES}

Chi, M. T. H., Bassock, M., Lewts, M. W., Reimann, P., \& GLASER, R. (1987). Self-explanations: How students study and use examples in leaming to solve problems (Tech. Rep. No. 9). Pitsburgh, PA: University of Pittsburgh, Leaming Research and Development Center. 
Chi, M. T. H., Feltovich, P. J., \&laser, R. (1981). Categorization and representation of physics problems by experts and novices. Cognitive Science, 5, 121-152.

Colluns, A., Brown, J. S., Newman, S. E. (in press). Cognitive apprenticeship: Teaching the crafts of reading, writing, and mathematics. In L. B. Resnick (Ed.), Knowing, leaming and instruction: Essays in honor of Robert Glaser. Hillsdale, NJ: Erlbaum.

EYLON, B. S., \& REIF, F. (1984). Effects of knowledge organization on task performance. Cognition \& Instruction, 1, 5-44.

Hayes, J. R., Simon, H. A. (1976). The understanding process: Problem isomorphs. Cognitive Psychology, 8, 165-190.

Heller, J. I., \& ReIF, F. (1984). Prescribing effective human problem solving processes: Problem description in physics. Cognition \& Instruction, 1, 177-216.

Hinsley, D. A., HAyes, J. R., \& Simon, H. A. (1977). From words to equations: Meaning and representation in algebra word problems. In M. A. Just \& P. A. Carpenter (Eds.), Cognitive processes in comprehension (pp. 89-106). Hillsdale, NJ: Erlbaum.

LARKIN, J. H. (1981). Enriching formal knowledge: A model for learning to solve problems in physics. In J. R. Anderson (Ed.), Cognitive skills and their acquisition (pp. 311-334). Hillsdale, NJ: Erlbaum.

LARKIN, J. H. (1983). The role of problem representation in physics. In D. Gentner \& A. L. Stevens (Eds.), Mental models (pp. 75-98). Hillsdale, NJ: Erlbaum.

Larkin, J. H., McDermott, J., Simon, D. P., \& Simon, H. A. (1980). Models of competence in solving physics problems. Cognitive Science, 4, 317-345.

Mervis, C. B. (1980). Category structure and the development of categorization. In R. J. Shapiro, B. C. Bruce, \& W. F. Brewer (Eds.), Theoretical issues in reading comprehension: Perspectives from cognitive psychology, linguistics, artificial intelligence and education (pp. 279307). Hillsdale, NJ: Erlbaum.

Mestre, J. P., Dufresne, R., Gerace, W., Hardiman, P. T., \& Touger, J. (1988). Promoting expert-like behavior among beginning physics students (Tech. Rep. No. 178). Amherst: Scientific Reasoning Research Institute, University of Massachusetts.

NEWELl, A., Simon, H. A. (1972). Human problem solving. Englewood Cliffs, NJ: Prentice-Hall.

NIEGEMANN, H., \&AAR, I. (1986). Classification of algebra and physics word problems by subjects of different levels of expertise (Tech. Rep. No. 33). Saarbrucken, Germany: Universität des Saarlandes, Fachrichtung Allgemeine Erziehungswissenschaft.

Resnick, R., \& Halldiay, D. (1977). Physics. New York: Wiley.

Rosch, E., \& Mervis, C. B. (1975). Family resemblances: Studies in the intemal structure of categories. Cognitive Psychology, 8, 382-439.

Schoenfeld, A. H., a Herrmann, D. J. (1982). Problem perception and knowledge structure in expert and novice mathematical problem solvers. Journal of Experimental Psychology: Leaming, Memory, \& Cognition, 8, 484-494.

SiLVER, E. A. (1979). Student perceptions of relatedness among mathematical verbal problems. Joumal for Research in Mathematics Education, 10, 195-210.

SIMON, D. P., SIMON, H. A. (1978). Individual differences in solving physics problems. In R. S. Sigler (Ed.), Children's thinking: What develops? (pp. 325-348). Hillsdale, NJ: Erlbaum.

SmgTH, E. E., SHOBEN, E. J., RIPS, L. J. (1974). Structure and process in semantic memory: A featural model for semantic decisions. Psychological Review, 81, 214-241.

\section{APPENDIX 1}

Model and Comparison Problems Used for Experiment 1

(Each item is composed of the model problem, with two comparison problems in one of the following combinations: $S-D$, S-SD, N-D, N-SD.)

\section{Model Problem 1}

A $90 \mathrm{~kg}$ mass is connected to a light horizontal spring of force constant $60 \mathrm{~N} / \mathrm{m}$ and placed on a surface with coefficient of static friction 0.4 . If the free end of the spring is slowly moved away from the mass, what distance may the free end be pulled before the mass begins to move?

\section{S Comparison Problem}

A $50 \mathrm{~kg}$ mass with an initial horizontal velocity of $5 \mathrm{~m} / \mathrm{sec}$ passes over a rough surface of length $0.5 \mathrm{~m}$ and coefficient of kinetic friction 0.2. After leaving the rough surface, it collides with a light horizontal spring of force constant $120 \mathrm{~N} / \mathrm{m}$. Find the maximum compression of the spring.

\section{Comparison Problem}

A $60 \mathrm{~kg}$ block is placed on a frictionless inclined plane of angle 25 degrees. The block is attached to a hanging mass by a light string over a frictionless pulley. Find the minimum value of the hanging mass so that the system remains in equilibrium.

\section{SD Comparison Problem}

A mass of $30 \mathrm{~kg}$ is hung from a light vertical spring of unstretched length $1 \mathrm{~m}$ and force constant $50 \mathrm{~N} / \mathrm{m}$. When the mass is in equilibrium, find the length of the spring.

\section{N Comparison Problem}

A $4 \mathrm{~kg}$ shell is fired with an initial velocity of $1,000 \mathrm{~m} / \mathrm{sec}$ from a cliff $120 \mathrm{~m}$ above level ground. What is its velocity when it hits the ground?

\section{Model Problem 2}

A $15 \mathrm{~kg}$ block and a $10 \mathrm{~kg}$ block are connected by a light compressed spring of force constant $200 \mathrm{~N} / \mathrm{m}$ and held at rest. The blocks are released and observed to move at $2 \mathrm{~m} / \mathrm{sec}$ and $3 \mathrm{~m} / \mathrm{sec}$, respectively, in opposite directions. Find the distance the spring was compressed from its equilibrium length.

\section{$S$ Comparison Problem}

Two blocks of mass $10 \mathrm{~kg}$ and $8 \mathrm{~kg}$ are connected by a light compressed spring of force constant $80 \mathrm{~N} / \mathrm{m}$ and held at rest. The blocks are released and the $10 \mathrm{~kg}$ block is observed to move at $2 \mathrm{~m} / \mathrm{sec}$. Find the velocity of the $8 \mathrm{~kg}$ block.

\section{Comparison Problem}

A $0.5 \mathrm{~kg}$ billiard ball of radius $2 \mathrm{~cm}$ rolls without slipping down an inclined plane. If the billiard ball is initially at rest, what is its speed after it has moved through a vertical distance of $0.5 \mathrm{~m}$ ?

\section{SD Comparison Problem}

A $50 \mathrm{~kg}$ mass with an initial horizontal velocity of $5 \mathrm{~m} / \mathrm{sec}$ passes over a rough surface of length $0.5 \mathrm{~m}$ and coefficient of kinetic friction 0.2 . After leaving the rough surface, it collides with a light horizontal spring of force constant $120 \mathrm{~N} / \mathrm{m}$. Find the maximum compression of the spring.

\section{N Comparison Problem}

A bullet of mass $10 \mathrm{~g}$ is fired at $800 \mathrm{~m} / \mathrm{sec}$ into a block of mass $10 \mathrm{~kg}$. If the bullet and block need $1 \mathrm{msec}$ to reach a common velocity, what is the average force exerted by the block on the bullet?

\section{Model Problem 3}

Two blocks of mass $10 \mathrm{~kg}$ and $8 \mathrm{~kg}$ are connected by a light compressed spring of force constant $80 \mathrm{~N} / \mathrm{m}$ and held at rest. The blocks are released and the $10 \mathrm{~kg}$ block is observed to move at $2 \mathrm{~m} / \mathrm{sec}$. Find the velocity of the $8 \mathrm{~kg}$ block.

\section{S Comparison Problem}

A $15 \mathrm{~kg}$ block and a $10 \mathrm{~kg}$ block are connected by a light compressed spring of force constant $200 \mathrm{~N} / \mathrm{m}$ and held at rest. The 
blocks are released and observed to move at $2 \mathrm{~m} / \mathrm{sec}$ and $3 \mathrm{~m} / \mathrm{sec}$, respectively, in opposite directions. Find the distance the spring was compressed from its equilibrium length.

\section{Comparison Problem}

A bullet of mass $10 \mathrm{~g}$ is fired at $800 \mathrm{~m} / \mathrm{sec}$ into a block of mass $10 \mathrm{~kg}$. If the bullet and block need $1 \mathrm{msec}$ to reach a common velocity, what is the average force exerted by the block on the bullet?

\section{SD Comparison Problem}

Two blocks each of mass $20 \mathrm{~kg}$ are connected by a light compressed spring of force constant $70 \mathrm{~N} / \mathrm{m}$. After the blocks are released, it is observed that one of the blocks has received an impulse of magnitude $150 \mathrm{~N} / \mathrm{sec}$. Find the speed of the other block.

\section{N Comparison Problem}

A $10 \mathrm{~kg}$ mass with initial velocity $2 \mathrm{~m} / \mathrm{sec}$ passes over a rough horizontal surface with a coefficient of kinetic friction 0.1 . Find the acceleration of the mass when it is on the rough surface.

\section{Model Problem 4}

A $2.5 \mathrm{~kg}$ ball of radius $4 \mathrm{~cm}$ is traveling at $7 \mathrm{~m} / \mathrm{sec}$ on a rough horizontal surface but not spinning. Some distance later, the ball is rolling without slipping at $5 \mathrm{~m} / \mathrm{sec}$. How much work was done by friction?

\section{S Comparison Problem}

A $3 \mathrm{~kg}$ soccer ball of radius $15 \mathrm{~cm}$ is initially sliding at $10 \mathrm{~m} / \mathrm{sec}$ without spinning. The ball travels on a rough horizontal surface and eventually rolls without slipping. Find the ball's final velocity.

\section{Comparison Problem}

A small rock of mass $10 \mathrm{~g}$ falling vertically hits a very thick layer of snow and penetrates $2 \mathrm{~m}$ before coming to rest. If the rock's speed was $25 \mathrm{~m} / \mathrm{sec}$ just prior to hitting the snow, find the average force exerted on the rock by the snow.

\section{SD Comparison Problem}

A $0.5 \mathrm{~kg}$ billiard ball of radius $2 \mathrm{~cm}$ rolls without slipping down an inclined plane. If the billiard ball is initially at rest, what is its speed after it has moved throught a vertical distance of $0.5 \mathrm{~m}$ ?

\section{N Comparison Problem}

A $2 \mathrm{~kg}$ projectile is fired with an initial velocity of $1,500 \mathrm{~m} / \mathrm{sec}$ at an angle of 30 degrees above the horizontal and height $100 \mathrm{~m}$ above level ground. Find the time needed for the projectile to reach the ground.

\section{Model Problem 5}

A $3 \mathrm{~kg}$ soccer ball of radius $15 \mathrm{~cm}$ is initially sliding at $10 \mathrm{~m} / \mathrm{sec}$ without spinning. The ball travels on a rough horizontal surface and eventually rolls without slipping. Find the ball's final velocity.

\section{S Comparison Problem}

A $0.5 \mathrm{~kg}$ billiard ball of radius $2 \mathrm{~cm}$ is initially sliding at $7 \mathrm{~m} / \mathrm{sec}$ on a rough horizontal surface without spinning. Some distance later, the ball is rolling without slipping at $5 \mathrm{~m} / \mathrm{sec}$. How much energy was lost due to friction?

\section{Comparison Problem}

A $20 \mathrm{~kg}$ stick of length $2 \mathrm{~m}$ is free to rotate about a vertical axle through one end on a frictionless horizontal surface. A $5 \mathrm{~g}$ bullet traveling perpendicular to the stick hits and embeds itself into the stick $50 \mathrm{~cm}$ from the pivot. If the initial speed of the bullet is $66 \mathrm{~m} / \mathrm{sec}$, what is the angular speed of the stick after the collision?

\section{SD Comparison Problem}

A billiard ball of radius $3 \mathrm{~cm}$ and mass $.2 \mathrm{~kg}$ is given a horizontal impulse of magnitude $.4 \mathrm{~N} / \mathrm{m}$ at a vertical distance of $1 \mathrm{~cm}$ from the top of the ball. If the ball rolls without slipping, find the final angular velocity of the billiard ball.

\section{N Comparison Problem}

A mass of $30 \mathrm{~kg}$ is hung from a light vertical spring of unstretched length $1 \mathrm{~m}$ and force constant $50 \mathrm{~N} / \mathrm{m}$. When the mass is in equilibrium, find the length of the spring.

\section{Model Problem 6}

A $20 \mathrm{~kg}$ stick of length $2 \mathrm{~m}$ is free to rotate about a vertical axle through one end on a frictionless horizontal surface. A $5 \mathrm{~g}$ bullet traveling perpendicular to the stick hits and embeds itself into the stick $50 \mathrm{~cm}$ from the pivot. If the initial speed of the bullet is $66 \mathrm{~m} / \mathrm{sec}$, what is the angular speed of the stick after the collision?

\section{S Comparison Problem}

A $1 \mathrm{~kg}$ stick of length $1 \mathrm{~m}$ is placed on a frictionless horizontal surface and is free to rotate about a vertical axle through one end. A $50 \mathrm{~g}$ lump of clay is attached $80 \mathrm{~cm}$ from the pivot. Find the frictional force between the stick and the clay when the angular velocity of the system is $3 \mathrm{rad} / \mathrm{sec}$.

\section{Comparison Problem}

A $3 \mathrm{~kg}$ soccer ball of radius $15 \mathrm{~cm}$ is initially sliding at $10 \mathrm{~m} / \mathrm{sec}$ without spinning. The ball travels on a rough horizontal surface and eventually rolls without slipping. Find the ball's final velocity.

\section{SD Comparison Problem}

A stick of length $1.5 \mathrm{~m}$ and mass $.2 \mathrm{~kg}$ is rotating about a pivot at one end on a frictionless surface. A $35 \mathrm{~g}$ lump of clay drops vertically onto the stick at its midpoint. If the clay remains attached to the stick, find the final angular velocity of the stick-clay system.

\section{N Comparison Problem}

A $2 \mathrm{~kg}$ block is at rest on a frictionless surface. A $30 \mathrm{~g}$ lump of putty sliding along the surface strikes and sticks to the $2 \mathrm{~kg}$ block. If the final speed of the block-putty system is $1 \mathrm{~m} / \mathrm{sec}$, what was the speed of the putty prior to the collision?

\section{Model Problem 7}

A bullet of mass $10 \mathrm{~g}$ is fired at $800 \mathrm{~m} / \mathrm{sec}$ into a block of mass $10 \mathrm{~kg}$. If the bullet and block need 1 msec to reach a common velocity, what is the average force exerted by the block on the bullet?

\section{S Comparison Problem}

A puck of mass $150 \mathrm{~g}$ traveling with speed $10 \mathrm{~m} / \mathrm{sec}$ in the positive $x$ direction collides with a second stationary puck of mass $200 \mathrm{~g}$. After the collision, the $150 \mathrm{~g}$ puck is observed to 
move in the negative $x$ direction with speed $1 \mathrm{~m} / \mathrm{sec}$. Find the velocity of the $200 \mathrm{~g}$ puck after the collision.

\section{Comparison Problem}

A $5 \mathrm{~g}$ bullet traveling with speed $300 \mathrm{~m} / \mathrm{sec}$ goes through a stationary wooden block of thickness $4 \mathrm{~cm}$. The bullet's speed after passing through the block is $250 \mathrm{~m} / \mathrm{sec}$. What was the average force exerted on the bullet by the block?

\section{SD Comparison Problem}

A $30 \mathrm{~g}$ lump of putty slides along a frictionless surface, strikes, and sticks to a $2 \mathrm{~kg}$ block. If the final velocity of the blockputty system is $1 \mathrm{~m} / \mathrm{sec}$, what was the speed of the putty prior to the collision?

\section{N Comparison Problem}

A $60 \mathrm{~kg}$ block is placed on a frictionless inclined plane of angle 25 degrees. The block is attached to a hanging mass by a light string over a frictionless pulley. Find the minimum value of the hanging mass so that the $60 \mathrm{~kg}$ block does not accelerate down the incline.

\section{Model Problem 8}

A $10 \mathrm{~kg}$ mass with initial velocity $2 \mathrm{~m} / \mathrm{sec}$ passes over a rough horizontal surface with a coefficient of kinetic friction 0.1 . Find the acceleration of the mass when it is on the rough surface.

\section{S Comparison Problem}

A $7 \mathrm{~kg}$ block is released from rest on an inclined plane with coefficient of kinetic friction .15. What is the speed of the block after it has moved through a vertical distance of $10 \mathrm{~cm}$ if the work done by friction is $.2 \mathrm{~J}$ ?

\section{Comparison Problem}

A $1 \mathrm{~kg}$ stick of length $1 \mathrm{~m}$ is placed on a frictionless horizontal surface and is free to rotate about a vertical axle through one end. A $50 \mathrm{~g}$ lump of clay is attached $80 \mathrm{~cm}$ from the pivot. Find the frictional force between the stick and the clay when the angular velocity of the system is $3 \mathrm{rad} / \mathrm{sec}$.

\section{SD Comparison Problem}

A $60 \mathrm{~kg}$ block is placed on an inclined plane of angle 30 degrees and coefficient of kinetic friction 0.1 . What is its acceleration as it slides down?

\section{N Comparison Problem}

A $2 \mathrm{~kg}$ block is at rest on a frictionless surface. A $30 \mathrm{~g}$ lump of putty sliding along the surface strikes and sticks to the $2 \mathrm{~kg}$ block. If the final speed of the block-putty system is $1 \mathrm{~m} / \mathrm{sec}$, what was the speed of the putty prior to the collision?

\section{APPENDIX 2 \\ Model and Comparison Problems used for Experiment 2 \\ (Each item was composed of the model problem and one com- parison problem.)}

\section{Model Problem 1}

A female orangutan (of weight $W$ ) sees that her infant (of weight $w$ ) is in danger on a branch a few yards away, below her. She grabs the nearest vine, swoops down and snatches the infant up precisely at the lowest point in her swing. If the mother orangutan had velocity $v$ just before she grabbed the infant, what was the velocity of the two orangutans just after they joined?

\section{S Comparison Problem}

A young boy, holding a ball of mass $M$, swings on a lightweight rope attached to a tree near his favorite swimming hole. At the bottom of the rope's swing, the child has a velocity $v$. If the boy drops the ball when he is 2 feet above the lowest point in the swing, what is his velocity just after he drops the ball? Take the boy's mass to be $m$ and the length of the rope to be $\ell$.

\section{Comparison Problem}

A rifle fires a bullet of mass $m$ into a block of mass $M$, resting on a frictionless surface. The bullet passes all the way through the block, and continues out the other side. If the final speed of the block is $V$, and that of the bullet is $v$, what was the speed of the bullet just before it hit the block?

\section{SD Comparison Problem}

A pendulum with a bob of mass $m$ is released from a height such that its velocity at the bottom of its swing is $v$. Just when the bob reaches this velocity, it collides and sticks to a stationary mass, $\boldsymbol{M}$. Find the velocity of the bob-mass system just after the collision.

\section{N Comparison Problem}

Two blocks, of equal mass $M$, on a level frictionless surface, are attached to each other by a string. If one block is pulled away from the other (such that the string is taut) with a force $F$, what is the tension in the string?

\section{Model Problem 2}

A block of mass $m$ is at rest on the floor. The block is connected to a light vertical spring of force constant $k$. If the free end of the spring is slowly pulled up, away from the block, what distance may the free end be moved before the block begins to move?

\section{S Comparison Problem}

A block of mass $m$ is dropped from a height $h$. The block lands on a light vertical spring of force constant $k$. If one end of the spring is attached to the floor, what is the maximum compression of the spring?

\section{Comparison Problem}

A $60 \mathrm{~kg}$ block is held in place on a frictionless inclined plane of angle 25 degrees. The block is attached to a hanging mass by a light string over a frictionless pulley. Find the minimum value of the hanging mass so that the block does not move when released.

\section{SD Comparison Problem}

A mass of $30 \mathrm{~kg}$ is hung from a light vertical spring of unstretched length $1 \mathrm{~m}$ and force constant $50 \mathrm{~N} / \mathrm{m}$. When the mass is in equilibrium, find the length of the spring.

\section{N Comparison Problem}

A $4 \mathrm{~kg}$ shell is fired with an initial velocity of $1,000 \mathrm{~m} / \mathrm{sec}$ from a cliff $120 \mathrm{~m}$ above level ground. What is its velocity when it hits the ground?

\section{Model Problem 3}

The mass $M$ of a pendulum of length $\ell$ is released from rest at a height $(1 / 2) \ell$ from the bottom of the pendulum's swing. What is the speed of the mass at its lowest point? Consider the rope to be massless. 


\section{S Comparison Problem}

The mass $M$ of a pendulum of length $\ell$ has a speed $v$ at the bottom of the pendulum swing. Find the tension in the rope at this point. Consider the rope to be massless.

\section{Comparison Problem}

A block is held at the top of an inclined plane of length $\ell$ and angle $\theta$. The block is then given an initial velocity $v$ down the plane. Assuming no friction between the block and the plane, find the kinetic energy of the block when it reaches the bottom of the plane.

\section{SD Comparison Problem}

If the bob of a pendulum of length $\ell$ has a speed $v$ at the bottom of its swing, how fast will it be going at the top of its swing, i.e. at height $2 \ell$ ?

\section{N Comparison Problem}

A cart of mass $M_{1}$ is rolling along a level surface at a speed $v$. A mass, $M_{2}$, is dropped from rest and lands on the cart. What is the final speed of the cart and the mass?

\section{Model Problem 4}

A block of mass $m$ on top of a vertical (massless) spring is pushed down, compressing the spring from its equilibrium position a distance $d$. The force constant of the spring is $k$. What distance above the compressed position will the block reach if the spring is released?

\section{S Comparison Problem}

A light spring with spring constant $k$ sits vertically, its bottom end attached to the floor. What sized mass would you need to put on the top end of the spring to compress to a fixed distance $d$ ?

\section{Comparison Problem}

The initial speed of a bullet fired horizontally at height $h$ is $v$. The bullet has mass $m$. Assuming there is no friction due to the air, what is the bullet's speed when it reaches a height $h / 2$ ?

\section{SD Comparison Problem}

A spring with spring constant $k$ is placed at the bottom of a frictionless inclined plane which makes an angle $\theta$ with the horizontal. A block is pressed against the spring downward along the plane until the spring is compressed a distance $x$. The block is then released. What is the velocity of the block when the spring reaches its uncompressed length?

\section{N Comparison Problem}

A bar of mass $M$ hangs from a ceiling via two light strings of equal length attached to the two ends. What is the tension in each string?

\section{Model Problem 5}

A $10 \mathrm{~kg}$ mass with initial velocity $2 \mathrm{~m} / \mathrm{sec}$ passes over a rough horizontal surface with a coefficient of kinetic friction 0.1 . Find the acceleration of the mass when it is on the rough surface.

\section{S Comparison Problem}

A $7 \mathrm{~kg}$ block with an initial velocity of $3 \mathrm{~m} / \mathrm{sec}$ passes over a rough horizontal surface with a coefficient of kinetic friction
1. Find the speed of the block after the work done by friction is $20 \mathrm{~J}$.

\section{Comparison Problem}

A $1 \mathrm{~kg}$ stick of length $1 \mathrm{~m}$ is placed on a frictionless horizontal surface and is free to rotate about a vertical axle through one end. A $50 \mathrm{~g}$ lump of clay is attached $80 \mathrm{~cm}$ from the pivot. Find the force that the stick exerts on the clay when the angular velocity of the system is $3 \mathrm{rad} / \mathrm{s}$.

\section{SD Comparison Problem}

A $60 \mathrm{~kg}$ block passes over a rough horizontal surface. The acceleration of the block is $2 \mathrm{~m} / \mathrm{sec}^{2}$ while on the rough surface. Find the coefficient of kinetic friction for the surface.

\section{N Comparison Problem}

A $2 \mathrm{~kg}$ block is at rest on a frictionless surface. A $30 \mathrm{~g}$ lump of putty sliding along the surface strikes and sticks to the $2 \mathrm{~kg}$ block. If the final speed of the block-putty system is $1 \mathrm{~m} / \mathrm{sec}$, what was the speed of the putty prior to the collision?

\section{Model Problem 6}

A $20 \mathrm{~kg}$ stick of length $2 \mathrm{~m}$ resting on a frictionless horizontal surface is free to rotate about a vertical axle through one end. A $5 \mathrm{~g}$ bullet traveling perpendicular to the stick hits and embeds itself into the stick $50 \mathrm{~cm}$ from the pivot. If the initial speed of the bullet is $66 \mathrm{~m} / \mathrm{sec}$, what is the angular speed of the stick immediately after the collision?

\section{S Comparison Problem}

A $1 \mathrm{~kg}$ stick of length $1 \mathrm{~m}$ is placed on a frictionless horizontal surface and is free to rotate about a vertical axle through one end. A $50 \mathrm{~g}$ lump of clay is attached $80 \mathrm{~cm}$ from the pivot. Find the force between the stick and the clay when the angular velocity of the system is $3 \mathrm{rad} / \mathrm{s}$.

\section{Comparison Problem}

An ice skater is rotating with an angular velocity $\omega$. By bringing in her arms, the skater is able to triple her rotation rate. By what factor did the skater's moment of inertia change?

\section{SD Comparison Problem}

A stick of length $1.5 \mathrm{~m}$ and mass $.2 \mathrm{~kg}$ on a frictionless horizontal surface is rotating about a pivot at one end. A $35 \mathrm{~g}$ lump of clay drops vertically onto the stick at its midpoint. If the clay remains attached to the stick, find the final angular velocity of the stick-clay system.

\section{N Comparison Problem}

In a game of shuffleboard, a disk is given an initial speed of $v$. It slides a distance $d$ before coming to rest. What is the coefficient of kinetic friction between the disk and the surface?

\section{Model Problem 7}

A $2.5 \mathrm{~kg}$ ball of radius $4 \mathrm{~cm}$ is sliding at $7 \mathrm{~m} / \mathrm{sec}$ on a rough horizontal surface, but not spinning. Some distance later, the ball is rolling without slipping at $5 \mathrm{~m} / \mathrm{sec}$. How much work was done by friction?

\section{S Comparison Problem}

A $6 \mathrm{~kg}$ bowling ball of radius $15 \mathrm{~cm}$ is initially sliding at $10 \mathrm{~m} / \mathrm{sec}$ without spinning. The ball travels on a rough horizontal 
surface and eventually rolls without slipping. Find the ball's final velocity.

\section{Comparison Problem}

A small rock of mass $10 \mathrm{gm}$ falling vertically hits a very thick layer of snow and penetrates $2 \mathrm{~m}$ before coming to rest. If the rock's speed was $25 \mathrm{~m} / \mathrm{sec}$ just prior to hitting the snow, find the average force exerted on the rock by the snow.

\section{SD Comparison Problem}

A $0.5 \mathrm{~kg}$ billiard ball of radius $2 \mathrm{~cm}$ rolls without slipping down an inclined plane. If the billiard ball is initially at rest, what is its speed after it has moved through a vertical distance of $0.5 \mathrm{~m}$ ?

\section{N Comparison Problem}

A $2 \mathrm{~kg}$ projectile is fired with an initial velocity of $1,500 \mathrm{~m} / \mathrm{sec}$ at an angle of 30 degrees above the horizontal and from height $100 \mathrm{~m}$ above level ground. Find the time needed for the projectile to reach the ground.

\section{Model Problem 8}

Two blocks of mass $10 \mathrm{~kg}$ and $8 \mathrm{~kg}$ are connected by a light compressed spring of force constant $80 \mathrm{~N} / \mathrm{m}$ and held at rest. The blocks are released and the $10 \mathrm{~kg}$ block is observed to move at $2 \mathrm{~m} / \mathrm{sec}$. Find the velocity of the $8 \mathrm{~kg}$ block.

\section{S Comparison Problem}

A $15 \mathrm{~kg}$ block and a $10 \mathrm{~kg}$ block are connected by a light compressed spring of force constant $200 \mathrm{~N} / \mathrm{m}$ and held at rest. The blocks are released and observed to move at $2 \mathrm{~m} / \mathrm{sec}$ and $3 \mathrm{~m} / \mathrm{sec}$ respectively in opposite directions. Find the distance the spring was compressed from its equilibrium length.

\section{Comparison Problem}

A bullet of mass $10 \mathrm{~g}$ is fired into a target of mass $10 \mathrm{~kg}$. The bullet and target then have a common velocity of $1 \mathrm{~m} / \mathrm{sec}$. How fast was the bullet moving just before it entered the target?

\section{SD Comparison Problem}

Two blocks each of mass $20 \mathrm{~kg}$ are connected by a light, compressed spring of force constant $70 \mathrm{~N} / \mathrm{m}$. After the blocks are released, it is observed that one of the blocks has received an impulse of magnitude $150 \mathrm{~N} / \mathrm{sec}$. Find the speed of the other block.

\section{N Comparison Problem}

A $10 \mathrm{~kg}$ mass with initial velocity $2 \mathrm{~m} / \mathrm{sec}$ passes over a rough horizontal surface with a coefficient of kinetic friction 0.1 . Find the acceleration of the mass when it is on the rough surface.

(Manuscript received August 10, 1987; revision accepted for publication January 23, 1989.) 